PACS: 72.20.Dp; 72.20.Fr; 72.20.My

\title{
Galvanomagnetic phenomena in HgMnTe and HgCdMnTe single crystals
}

\author{
S.E. Ostapov, I.M. Rarenko, M.D. Tymochko \\ Chernivtsi National University, 2, Kotsyubinsky str., 58012 Chernivtsi, Ukraine
}

\begin{abstract}
This paper presents theoretical and experimental investigations of narrow-gap semiconductors $\mathrm{HgMnTe}$ and $\mathrm{HgCdMnTe}$. It has been shown that the comparison of temperature dependencies of the conductivity and Hall coefficient in the mixed conductivity region makes it possible to determine the content of cadmium and manganese, as well as the concentration of doping acceptor impurity.
\end{abstract}

Keywords: semiconductors, electronic transport, galvanomagnetic effects.

Manuscript received: 30.06.04; accepted for publication 16.12.04.

Today $\mathrm{Hg}_{1-\mathrm{x}} \mathrm{Cd}_{\mathrm{x}} \mathrm{Te}$ is a basic semiconductor material for manufacturing IR radiation detectors over a wide spectral range $(1 \ldots 14 \mu \mathrm{m})$, especially in a longwave window $8 \ldots 14 \mu \mathrm{m}$. The reason is that this material possesses a number of unique physical properties, such as a smooth dependency of the energy gap on chemical composition, small effective mass and high mobility of electrons, possibility of changes in the carrier concentration of both $p$ - and $n$ - conductivity types over the wide range $\left(10^{14}<n, p<10^{18} \mathrm{~cm}^{-3}\right)$ by technological methods [1].

At the same time, $\mathrm{Hg}_{1-\mathrm{x}} \mathrm{Cd}_{\mathrm{x}} \mathrm{Te}$ has a variety of serious shortcomings. First, it is a crystal lattice temporal instability caused by a weak bond of mercury atoms in the lattice. This results in formation of electrically active mercury vacancies - acceptors, leading to the redistribution of the level of electrically active current carrier centers [2,3]. Second, it is considerable dependency of bulk and surface material properties on the crystal structure perfection of $\mathrm{Hg}_{1-\mathrm{x}} \mathrm{Cd}_{\mathrm{x}}$ Te crystals.

Investigations have been underway for more than 20 years to find the possibilities of using alternative materials to HgCdTe. The most widespread in this respect are HgMnTe and HgCdMnTe [4]. The encouraging results have been obtained concerning the basic parameters of such detectors, especially detectivity acceptable for practical use. At the same time, the scientists encountered a series of problems, the basic one of which is the parameter non-reproducibility of detectors made of the same $\mathrm{HgMnTe}$ or $\mathrm{HgCdMnTe}$ crystal ingot. Temperature dependencies of the electric conductivity and Hall coefficient in this case have not only quantitative, but also qualitative distinctions.

This paper is an attempt to generalize the experimental results and provide them with theoretical substantiation. The crystals to be studied were made by the modified zone melting method. The content of manganese and cadmium in the synthesis was assigned in such a manner that the energy gap of material at the liquid nitrogen temperature should be $0.1 \mathrm{eV}$.

Fig. 1 represents the temperature dependency of the conductivity and Hall coefficient for two samples of $\mathrm{HgCdMnTe}$ and one - HgMnTe. As is seen from the figures, temperature dependencies have not only quantitative, but also qualitative distinctions for $\mathrm{HgCdMnTe}$ samples cut from the same ingot. It is reasonable to suppose that due to such qualitative distinctions in the curves, one can obtain specific information on material from the experimental dependencies of the conductivity and Hall effect, comparing them to theoretical calculations for various material parameters.

The resulting experimental data can be explained in terms of the mixed conductivity model for narrow-gap semiconductor with regard to a drastic difference in electron and hole mobility.

The energy gap was calculated by a simple method, when an arbitrary band parameter of quarternary material is represented as a combination of the appropriate values of two ternary materials $[5,6]$. In particular, for the energy gap:

$$
\begin{aligned}
& E_{g}\left(\operatorname{Hg}_{1-\mathrm{x}-\mathrm{y}} \mathrm{Cd}_{\mathrm{x}} \mathrm{Mn}_{\mathrm{y}} \mathrm{Te}\right)= \\
& =0.5 E_{g}\left(\mathrm{Hg}_{1-2 \mathrm{x}} \mathrm{Cd}_{2 \mathrm{x}} \mathrm{Te}\right)+ \\
& +0.5 E_{g}\left(\mathrm{Hg}_{1-2 \mathrm{y}} \mathrm{Mn}_{2 \mathrm{y}} \mathrm{Te}\right) .
\end{aligned}
$$

The peculiarity of this method lies in the fact that in contrast to the Williams method [7], it allows deriving a rather simple empirical formula for semiconductor energy gap.

The charge carrier concentration was calculated from solving the electric neutrality equation with regard for the possibility of electron degeneration. In this case, the electron and hole concentrations was assigned by the expressions: 


$$
\begin{aligned}
& p=\frac{N_{v}}{\exp \left(\frac{\Delta \mu}{k T}\right)+1}, \\
& n=\int_{0}^{\infty} \frac{N_{c}(E)}{\exp \left(\frac{E_{g}-\Delta \mu}{k T}\right)+1} d E,
\end{aligned}
$$

where $\Delta \mu$ is the Fermi level position (from the boundary of the valence band); $N_{v}=2\left(m_{h} k T / 2 \pi \hbar^{2}\right)^{3 / 2}$ is the density of states in the valence band; $N_{c}(E)$ is the density of states in the conductivity band which in the Kane approximation is of the following form:

$$
N_{c}(E)=\frac{\sqrt{2} m_{e}^{3 / 2}}{\pi^{2} \hbar^{3}} E^{1 / 2}\left(1+\frac{E}{E_{g}}\right)^{1 / 2}\left(1+2 \frac{E}{E_{g}}\right),
$$

the effective mass of electrons being determined in the same way as the energy gap, and the matrix element of the Kane model being assumed as equal to $P=8.1 \cdot 10^{-8} \mathrm{eV} \cdot \mathrm{cm}[8]$.

In calculations of the mobility, we took four mechanisms of scattering into account: on composition fluctuations, ionized impurities, polar optical and acoustic phonons [9].

The scattering on the fluctuations of composition was taken into account with the relaxation time [10]:

$$
\frac{1}{\tau_{\mathrm{fl}}(\varepsilon)}=\frac{\pi x(1-x)}{\hbar N_{0}}\left(\frac{\partial E_{V}}{\partial x}\right)^{2} N_{h h}(\varepsilon),
$$
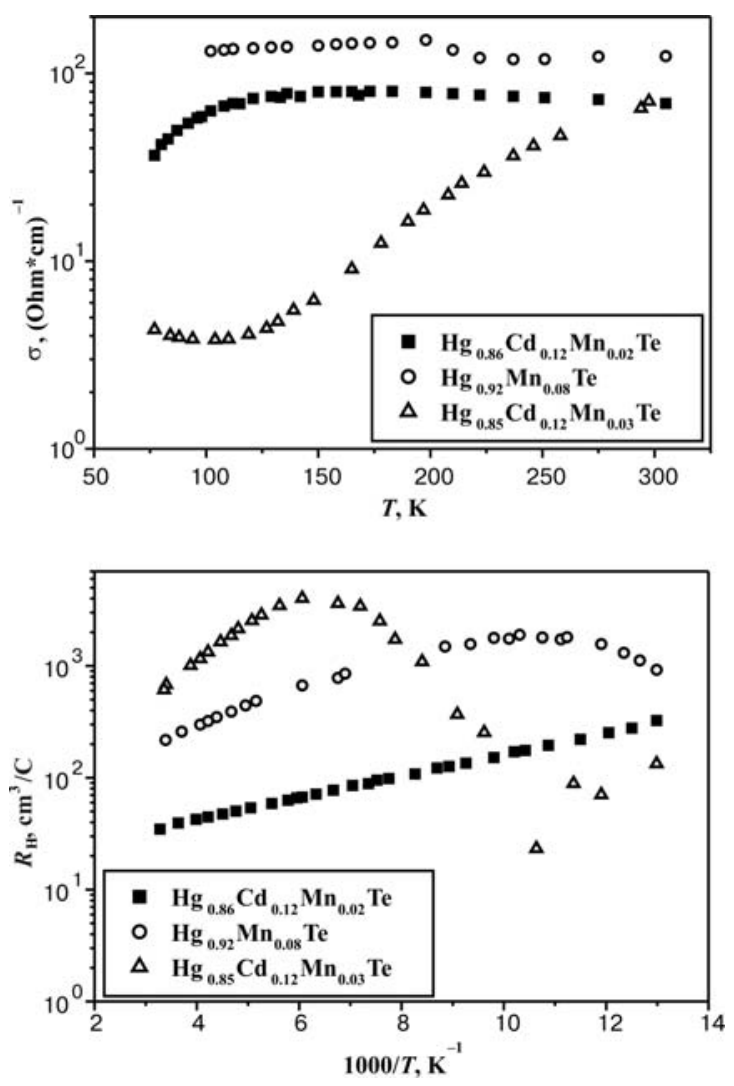

Fig. 1. Temperature dependencies of conductivity and Hall coefficient in HgMnTe and $\mathrm{HgCdMnTe}$. where $N_{0}$ is the concentration of atoms in the metal sublattice; $N_{h h}(\varepsilon)$ is the dispersion law of heavy holes, $d E_{V} / d x$ is the shift of the valence band edge.

Though scattering by ionized impurities in crystals under study is predominant at $T<80 \mathrm{~K}$, it should be taken into account at higher temperatures as well. The relaxation time for this scattering mechanism can be calculated by the formula [9]:

$\tau_{\text {ion }}=\left(\varepsilon_{\mathrm{s}} \hbar^{3 / 2} \pi e^{4} N_{i}\right)\left(k^{3} / m^{*}\right)\left(1 / F_{\text {ion }}\right)$

where $F_{\text {ion }}$ is a function taking into account the symmetry of electron wavefunctions and shielding of the scattering centre potential with free carriers; $N_{i}$ is the concentration of ionized impurities; $\varepsilon_{s}$ is the sum of static and high-frequency dielectric constants. The rest of designations are generally accepted.

The scattering on optical and acoustic phonons in $\mathrm{Hg}_{1-\mathrm{x}-\mathrm{y}} \mathrm{Cd}_{\mathrm{x}} \mathrm{Mn}_{\mathrm{y}} \mathrm{Te}$ crystals (like in $\mathrm{Hg}_{1-\mathrm{x}} \mathrm{Cd}_{\mathrm{x}} \mathrm{Te}$ ) is predominant at the temperatures above $80 \mathrm{~K}$, especially in the area of intrinsic conductivity. In the case of the carrier scattering by polar optical phonons, the expression for the relaxation time is of the following form:

$\tau_{\mathrm{op}}=\left(\varepsilon_{\mathrm{o}} \hbar / 2 e^{2} k_{0} T\right)(\partial E / \partial k) 1 / F_{\mathrm{op}}$,

and with the scattering on acoustic lattice vibrations

$\tau_{\mathrm{ac}}=\left(\pi \hbar \rho v^{2} / E_{0}{ }^{2} k_{0} T F_{\mathrm{ac}}\right)(\partial E / \partial k) 1 / k^{2}$.

In Eqs (7) and (8), $\rho$ is the crystal density, $E_{0}$ is the acoustic deformation potential constant, $F_{\text {ac }}$ and $F_{\text {op }}$ are the functions that take into account the influence of the Bloch factors in the Kane model for acoustic and polar optical phonons.

Fig. 2 represents the contribution of various scattering mechanisms to the mobility and a comparison to experiments.

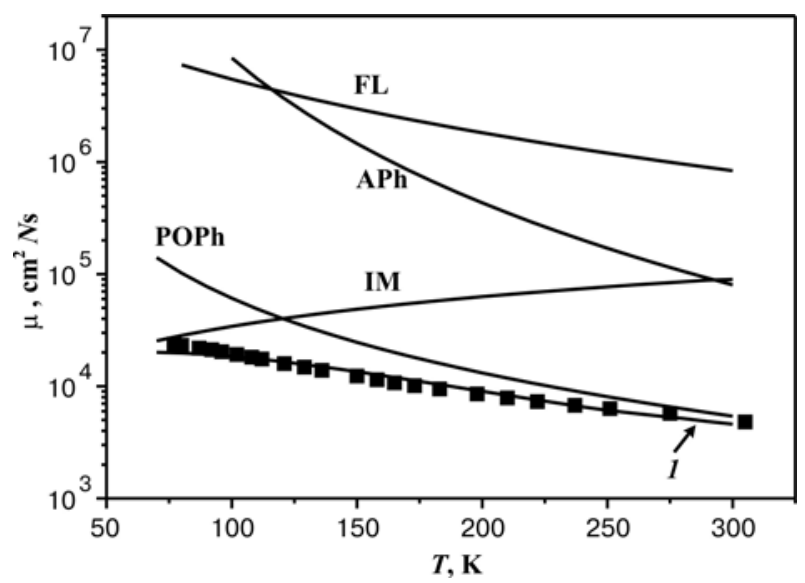

Fig. 2. Mobility temperature dependency in $\mathrm{HgCdMnTe}$. Solid curves: $\mathrm{APh}$ - calculated mobility, taking acoustic phonons into account; IM - ionized impurities; POPh - polar optic phonons, FL - composition fluctuations. Curve 1 - total mobility. 
As is seen from the figure, $\mathrm{HgCdMnTe}$ mobility is almost completely determined by the scattering by polar optical phonons (POPh) and ionized impurities (IM), which is in agreement with the results reported in Ref. $[9,10]$. At low temperatures, the ionized impurities and polar optical phonons (at given impurity concentrations) determine relevant scattering mechanisms. With the increase in temperature, the latter become much more important, whereas the contribution of ionized impurities becomes ever-decreasing. Finally, at room temperatures, the mobility is almost completely determined by polar optical phonons. It is obvious that as the concentration of impurities increases their contribution to scattering will grow, curve IM will be lowered, leading to mobility reduction. At the same time, the role of acoustic phonons (APh) becomes more essential, so curve 1 (total mobility) is more and more deflected from the $\mathrm{POPh}$ curve. One can see that the scattering on the composition fluctuations is not important, which is in agreement with the results in [11].

As it is seen from Fig. 2, the mobility calculated in this approximation is in a good agreement with experimental data for $\mathrm{HgCdMnTe}$ (the same is observed for HgMnTe, see Ref. [12]), testifying to the similarity of materials and mechanisms of charge carrier scattering therein.
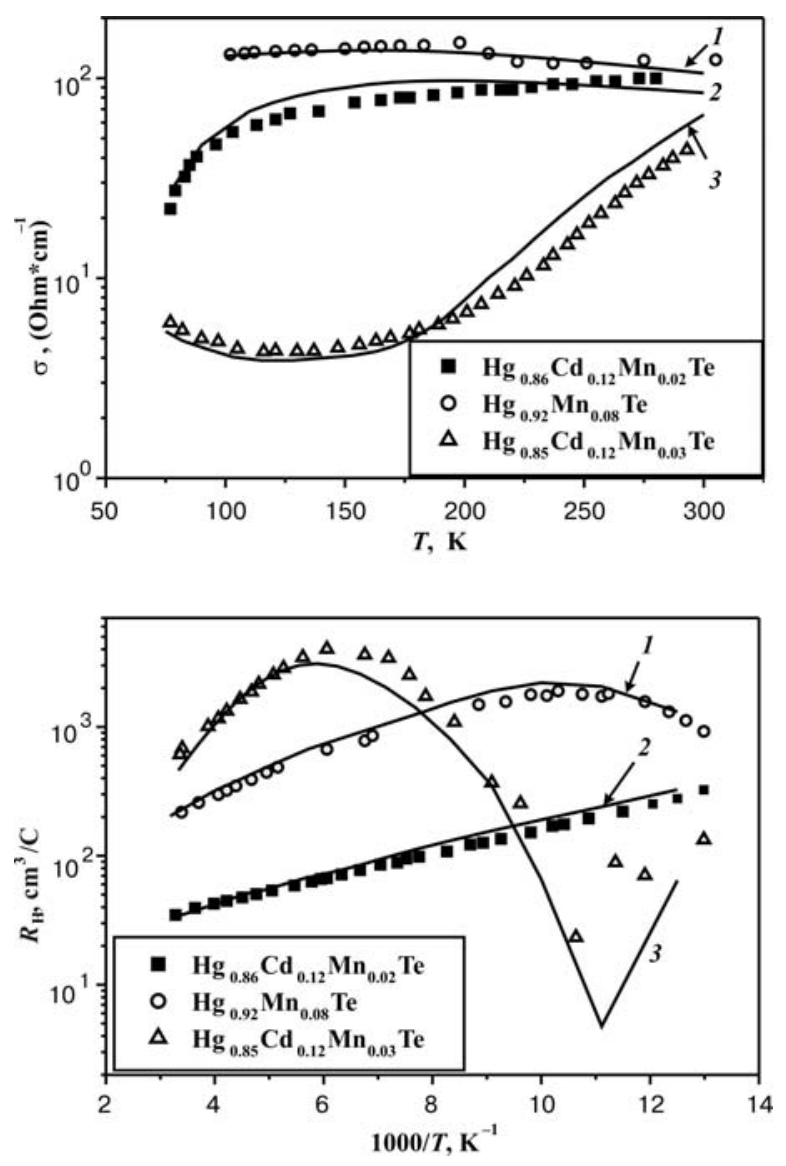

Fig. 3. The temperature dependencies of conductivity and Hall coefficients in HgMnTe and $\mathrm{HgCdMnTe}$. Curve $1-\mathrm{HgMnTe}$ $\left(N_{a}=610^{16} \mathrm{~cm}^{-3}\right) ; 2-\operatorname{HgCdMnTe}\left(N_{a}=6 \cdot 10^{15} \mathrm{~cm}^{-3}\right) ; 3-$ HgCdMnTe with $N_{a}=10^{17} \mathrm{~cm}^{-3}$. Donor concentration $N_{d}=10^{15} \mathrm{~cm}^{-3}$.
The results of calculations of the conductivity and Hall coefficient with regard for all these three scattering mechanisms are shown in Fig. 3.

It is seen that the agreement between theoretical calculations and experimental data is quite satisfactory in all the cases. Despite this different behaviour of dependencies, all of them, as we see, fit to the mixed conductivity model both for $\mathrm{HgMnTe}$ and $\mathrm{HgCdMnTe}$.

Extraordinary sensitivity of $\sigma(T)$ and $R_{\mathrm{H}}(T)$ to a change in the material composition and doping impurity concentration is conspicuous. Thus, curves 1 describe almost zero-gap HgMnTe $(x=0.075) \quad$ with $N_{a}=6 \cdot 10^{16} \mathrm{~cm}^{-3}$. With this concentration of acceptors, when all of them are fully ionized, they determine the behavior of kinetic coefficients almost entirely. The same conclusions can be made about curves 3 ( $\mathrm{HgCdMnTe})$, where acceptor concentration is even higher $\left(1 \cdot 10^{17} \mathrm{~cm}^{-3}\right)$, but the energy gap is considerably larger (the sample composition is $x=0.12, y=0.03$, corresponding to $\mathrm{HgCdTe}$ of composition $x \approx 0.18$ ). This is a material with pronounced $p$-type of conductivity. In $\sigma(T)$ dependency, one can clearly see the minimum in the vicinity of $100 \mathrm{~K}$, and $R_{\mathrm{H}}(T)$ demonstrates a distinct change of its sign.

Curve 2 shows material $(x=0.12, y=0.02)$ of weak $p$-type $\left(N_{a}=6 \cdot 10^{15} \mathrm{~cm}^{-3}\right)$. Due to a strong influence of electrons, we have almost monotonous dependencies.

As it was supposed, the donor concentration in all the cases considered above is of no fundamental importance as long as it is less than the acceptor concentration. This fact is observed both in $\mathrm{HgCdMnTe}$ and $\mathrm{HgMnTe}$ [12]. Emphasize again a strong sensitivity of $\sigma(T)$ and $R_{\mathrm{H}}(T)$ to a change in material composition, which is exhibited not only in quantitative, but also in qualitative changes of $\sigma(T)$ and $R_{\mathrm{H}}(T)$ dependencies. It allows determining the precisely enough material composition and doping degree based on the analysis of material temperature dependencies.

Note that this identification is only possible when the behaviour of $\sigma(T)$ and $R_{\mathrm{H}}(T)$ is strongly affected by the electron component. For example, in the case of $\mathrm{Hg}_{1-\mathrm{x}} \mathrm{Mn}_{\mathrm{x}} \mathrm{Te}(x=0.3)$, when the energy gap at $300 \mathrm{~K}$ $E_{g} \approx 0.7 \mathrm{eV}$, with temperature reduction for any acceptor concentration there is a monotonous increase in conductivity. The above analysis is equally impossible for $n$-type material, because in this case the mixed conductivity is not observed for any sample compositions whatsoever due to a large difference between electron and hole mobilities.

Thus, as a result of the investigations the following conclusions can be made:

- In the formation of temperature dependencies of mobility in zero-gap and narrow-gap HgMnTe and $\mathrm{HgCdMnTe}$ crystals of basic importance are three scattering mechanisms: ionized impurities, polar optical and acoustic phonons.

- The scattering on the composition fluctuations is not important in the temperature range $80 \ldots 300 \mathrm{~K}$.

- Comparison of temperature dependencies of the conductivity and Hall coefficient makes it possible 
to determine the content of cadmium and manganese, as well as the concentration of doping acceptor impurity.

\section{Acknowledgements}

This work was financially supported by Science and Technology Center of Ukraine (STCU) under Project \#1440.

\section{References}

1. R. Dornhaus, G. Nimtz, The properties and applications of the HgCdTe alloy system // Springer Pract. Mod. Phys., Berlin, 98, p. 119-304 (1983).

2. H.R. Vydynath, Lattice defects in narrow-gap semiconductors $\mathrm{HgCdTe}$ alloys // J. Electrochem. Soc. p. 2609-2619 (1981).

3. G. Nimtz, B. Schlicht, R. Dornhaus, Long-term Hall-type conversion by vacancy diffusion in $\mathrm{HgCdTe}$ at room temperature // Appl. Phys. Lett. 34 (8), p. 490-491 (1979).

4. A. Rogalski, $\mathrm{Hg}_{1-x} \mathrm{Mn}_{x} \mathrm{Te}$ as a new infrared detector material // Infrared Phys. 31, p. 117-166 (1991).

5. O.A. Bodnaruk, A.V. Markov, S.E. Ostapov, I.M. Rarenko, A.F. Slonetski, Bandgap and intrinsic carrier concentration in $\mathrm{HgCdMnTe}$ and $\mathrm{HgCdZnTe}$ // Semiconductors 34, p. 415-417 (2000).
6. O.A. Bodnaruk, A.V. Markov, S.E. Ostapov, 11th Intern. Conf. on Ternary \& Multinary Compounds 8-12.09.1997, Salford, UK, Abstracts, p. P1-74.

7. C.K. Williams, T.H. Glisson, J.R. Hauser and M.A. Littlejohn, Energy bandgap and lattice constant contours of III-V quaternary alloys of the form $\mathrm{A}_{X} \mathrm{~B}_{Y} \mathrm{C}_{Z} \mathrm{D}$ or $\mathrm{AB}_{X} \mathrm{C}_{Y} \mathrm{D}_{Z} / /$ J. Electron. Mater. 7, p. 639-646 (1978).

8. G.V. Beketov, A.E. Belyaev et al., The peculiarities of transport phenomena in epitaxial layers MnHgTe/CdZnTe // Fiz. Tekhn. Poluprov. 31 (3), p. 268-272 (1997).

9. A.I. Vlasenko, Ya.M. Olikh, and R.K. Savkina, Charge carrier mobility in $n$-CdHgTe crystals subjected to dynamic ultrasonic stressing // Semiconductors 34 (6), p. 644-649 (2000).

10. V.V. Bogoboyashchyy, About holes scattering mechanisms in p-HgCdTe at low temperatures // Fiz. Tekhn. Poluprov. 36 (12), p. 1418-1425 (2002).

11. I.M. Nesmelova, The optical properties of narrowgap semiconductors. Novosibirsk, Nauka (1992).

12. L.A. Kosyachenko, A.V. Markov, S.E. Ostapov, I.M. Rarenko, V.M. Sklyarchuk, E.F. Sklyarchuk, The investigation of physical properties of p-type $\mathrm{HgMnTe}$ in the mixed conductivity region // $\mathrm{J}$. Phys. Study 7 (1), p. 365-369 (2003). 